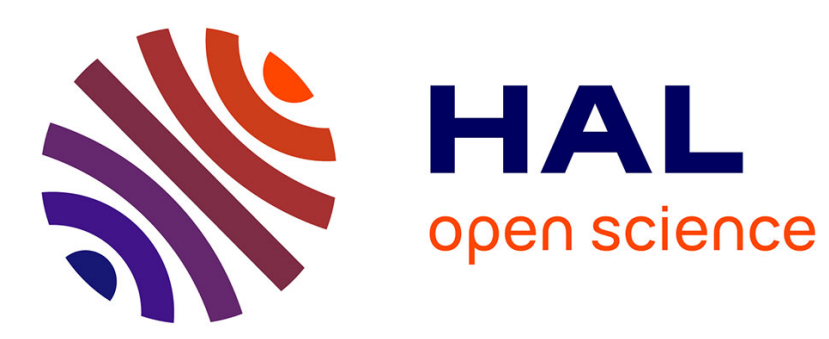

\title{
Structure et propriétés électroniques de très petits agrégats de lithium dans une matrice de $\mathrm{MgO}$
}

\author{
A. Julg, M. Bourg
}

\section{To cite this version:}

A. Julg, M. Bourg. Structure et propriétés électroniques de très petits agrégats de lithium dans une matrice de MgO. Journal de Physique Lettres, 1982, 43 (7), pp.243-247. 10.1051/jphyslet:01982004307024300 . jpa-00232040

\section{HAL Id: jpa-00232040 https://hal.science/jpa-00232040}

Submitted on 1 Jan 1982

HAL is a multi-disciplinary open access archive for the deposit and dissemination of scientific research documents, whether they are published or not. The documents may come from teaching and research institutions in France or abroad, or from public or private research centers.
L'archive ouverte pluridisciplinaire HAL, est destinée au dépôt et à la diffusion de documents scientifiques de niveau recherche, publiés ou non, émanant des établissements d'enseignement et de recherche français ou étrangers, des laboratoires publics ou privés. 
Classification

Physics Abstracts

36.40

\title{
Structure et propriétés électroniques de très petits agrégats de lithium dans une matrice de MgO
}

\author{
A. Julg $(*)$ et M. Bourg $(* *)$ \\ Université de Provence, Place V. Hugo, 13331 Marseille Cedex 3, France \\ (Reçu le 16 décembre 1981, révisé le ler février 1982, accepté le 8 février 1982)
}

\begin{abstract}
Résumé. - Nous avons calculé par la méthode du champ moléculaire self-consistent la structure électronique de très petits agrégats de lithium inclus dans une matrice de $\mathrm{MgO}$. Les niveaux électroniques des agrégats dans la matrice sont abaissés par rapport à ceux des agrégats correspondants isolés. L'effet de la charge totale des ions enlevés pour former la cavité est examiné.

Abstract. - We have calculated by means of the molecular self-consistent method the electronic structure of minute aggregates of lithium, located in an $\mathrm{MgO}$ matrix. The electronic levels of the aggregates in the matrix are lowered with respect to those corresponding to the isolated aggregates. The total charge effect of the ions removed to form the cavity is examined.
\end{abstract}

Introduction. - L'utilisation de particules immergées dans un milieu diélectrique a trouvé un renouveau dans la recherche d'absorbeurs sélectifs liés à l'utilisation de l'énergie solaire. Les particules utilisées sont de l'ordre du micron, c'est-à-dire qu'elles contiennent un nombre d'atomes de l'ordre de $10^{9}$. L'étude des propriétés optiques, tant du point de vue théorique que du point de vue expérimental, de ces cermets a été faite depuis plusieurs années [1].

Il était intéressant de se demander, malgré la différence des échelles de taille, ce qui se passerait lorsque la dimension de ces agrégats diminuerait jusqu'à ne comprendre que quelques atomes. Il faut, pour cela, calculer leur structure électronique, c'est le but de ce travail. Les calculs ont été effectués pour de tout petits agrégats de deux à huit atomes de lithium immergés dans un réseau C.F.C. de charges alternativement positives et négatives représentant un cristal de $\mathrm{MgO}$.

1. Méthode d'étude. - Dans l'impossibilité de traiter directement le système (cristal lacunaire plus atomes inclus) dans son ensemble, des approximations sont nécessaires. Profitant de la forte localisation des charges électroniques autour des noyaux de $\mathrm{Mg}$ et de $\mathrm{O}$ du réseau, le problème peut être ramené à celui de petits agrégats de $\mathrm{Li}_{n}$ dans le champ d'un réseau de charges ponctuelles. Cette simplification permet alors de faire appel aux méthodes traditionnelles de la Chimie Quantique. Pratiquement, nous avons utilisé un programme de calcul self-consistent ab initio qui prend pour base des orbitales atomiques de Slater (Slater type orbitals) simulées par six fonctions gaussiennes, programme connu sous le signe GAUSSIAN-70, STO-6G [2]. La version employée permet l'introduction de plusieurs centaines de charges ponctuelles autour de la molécule considérée [3].

$\left(^{*}\right)$ Laboratoire de Chimie Théorique.

(**) Laboratoire de Physique Expérimentale. 
Des études préliminaires [4], faites sur la convergence du potentiel électrostatique à l'intérieur d'une cavité dans un cristal ionique de symétrie cubique, nous ayant montré que quelques centaines de charges ponctuelles disposées aux nœuds du réseau inscrit dans un cube, permettent de reproduire avec une excellente précision le champ électrostatique à l'intérieur de la cavité, nous avons introduit les charges situées à l'intérieur d'un cube contenant 8 charges par arête, la lacune destinée à recevoir les atomes de lithium étant située au centre.

Dans les calculs présentés ici, nous avons adopté, pour valeur des charges, celles correspondant aux ions, c'est-à-dire +2 et -2 . En fait, il semble bien que les charges nettes portées par les divers noyaux soient légèrement inférieures en valeur absolue, de l'ordre de 1,8 [5]. Les effets obtenus devraient donc être systématiquement surestimés de $10 \%$ environ.

Parmi les grandeurs que permet d'atteindre le programme utilisé, nous retiendrons la valeur des énergies $e_{i}$ associées aux divers niveaux électroniques $i$. En effet, l'opposé de $e_{i}$ (théorème de Koopmans [6]) donne l'énergie d'arrachement d'un électron du niveau correspondant soit, pour le niveau occupé le plus haut HOMO (Highest Occupied Molecular Orbital), le travail de sortie dans le cas d'un cristal. Une valeur positive pour HOMO signifie que l'on est sur un niveau antiliant, il y a donc départ spontané de l'électron.

L'énergie mise en jeu dans les transitions électroniques fait intervenir les $e_{i}$. Par exemple, pour une transition monoélectronique singulet-singulet entre le niveau initial doublement utilisé $\underline{i}$ et le niveau vide $j$, l'énergie mise en jeu est :

$$
{ }^{\mathrm{s}} E_{i \rightarrow j}=\left(e_{i}-e_{j}\right)-\left(J_{i j}-2 K_{i j}\right)
$$

où $J_{i j}$ et $K_{i j}$ sont respectivement les intégrales coulombiennes et d'échange relatives aux fonctions moléculaires associées aux niveaux $\underline{i}$ et $\underline{j}$. Ainsi, pour la molécule $\mathrm{Li}_{2}$ isolée, nous obtenons, pour la transition :

$$
{ }^{1} \Sigma_{\mathrm{g}}^{+} \rightarrow{ }^{1} \Sigma_{\mathrm{u}}^{+}: 6,3-4,0=2,3 \mathrm{eV}
$$

Cette valeur est peu différente de celles obtenues par d'autres auteurs avec des bases d'orbitales atomiques un peu différentes : 2,0 à 2,1 eV [7]. Expérimentalement la valeur observée est de l'ordre de $1,8 \mathrm{eV}$ [7]. La surestimation des énergies de transition dans ce type de méthode est générale. Elle provient du fait que la méthode SCF classique n'introduit pas la corrélation entre les électrons. L'interaction de configuration qui permet de tenir compte de cette dernière apporte d'excellents résultats [8]. Le volume des calculs est considérablement augmenté. Etant donné, d'une part que, plus que l'énergie exacte de la transition, c'est le déplacement spectral provoqué par la présence du réseau extérieur qui nous intéresse, et que, d'autre part, pour une molécule donnée, dans un état donné, l'énergie de corrélation ne dépend pratiquement que du nombre d'électrons dans la molécule, pour obtenir le déplacement spectral, il est inutile d'introduire la corrélation dans les calculs.

Par ailleurs, nous avons vérifié sur les calculs numériques que le terme $(J-2 K)$ pour une molécule enrobée n'est significativement pas différent de celui relatif à la molécule isolée, de sorte que, pratiquement, le déplacement spectral proviendra uniquement de la différence $\left(e_{j}-e_{i}\right)=\Delta e_{i j}$

2. Résultats des calculs. - Pour loger l'agrégat $\mathrm{Li}_{n}$, on a pratiqué, dans le réseau de charges, une cavité de volume suffisant en enlevant un nombre égal de charges positives et de charges négatives, c'est la cavité qualifiée de « neutre ». Dans d'autres cas on a, en outre, enlevé 2 ou 4 charges positives ou négatives. Dans le cas de $\mathrm{Li}_{2}$, on a essayé plusieurs positions de la cavité selon un axe 2 et selon un axe 4 du cube des charges. On a également pratiqué une cavité plus ou moins volumineuse pour $\mathrm{Li}_{2}$ et pour une direction donnée.

Les graphiques de la figure 1 résument les résultats des calculs. Les niveaux de cœur n'ont pas 


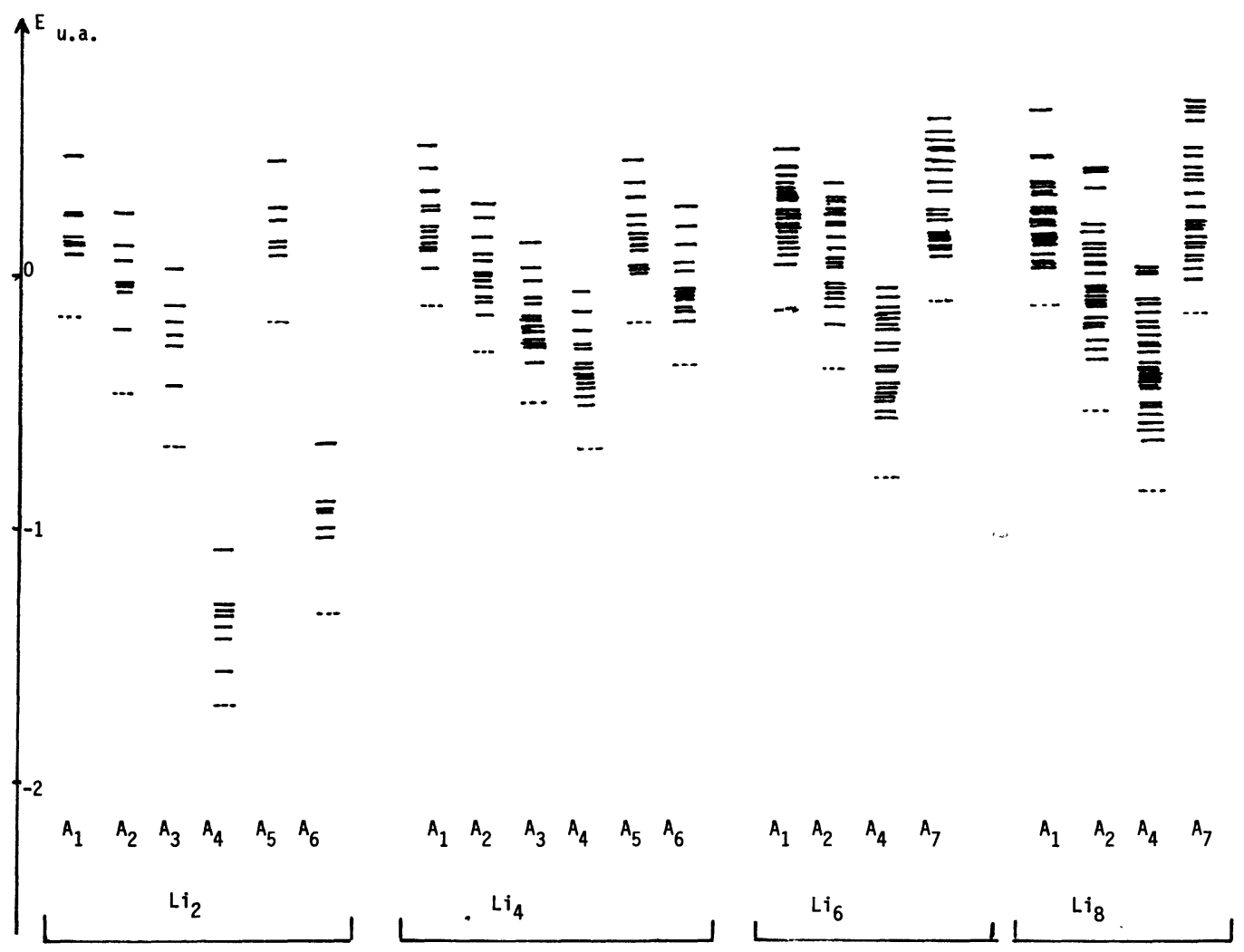

Fig. 1. - Colonne $A_{1}$ : agrégat isolé. $A_{2}$ : agrégat dans une cavité « neutre ». $A_{3}$ : agrégat dans une cavité où on a enlevé 2 charges - . $A_{4}$ : agrégat dans une cavité où on a enlevé 4 charges - . $A_{5}$ : agrégat chargé $2-$ dans une cavité où on a enlevé 2 charges.$- A_{6}$ : agrégat chargé 2 - dans une cavité où on a enlevé 4 charges -. $A_{7}$ : agrégat chargé 4 - dans une cavité où on a enlevé 4 charges - . Le niveau HOMO est en pointillé.

[Column $A_{1}$ : isolated aggregate. $A_{2}$ : aggregate in a "neutral » cavity. $A_{3}:$ aggregate in a cavity where two negative charges have been removed. $A_{4}$ : aggregate in a cavity where four negative charges have been removed. $A_{5}$ : two negative charged aggregate - in a cavity where two charges have been removed. $A_{6}:$ two negative charged aggregate - in a cavity where four charges have been removed. $A_{7}$ : four negative charged aggregate - in a cavity where four charges have been removed. The HOMO level is in a dotted line.]

été représentés pour des motifs de dimension de figure; ils sont de peu d'intérêt pour les propriétés physiques; nous avons constaté qu'ils suivent, avec une amplitude plus faible, les variations observées sur les niveaux supérieurs.

On constate que :

1) L'introduction de l'agrégat dans la cavité fait descendre l'ensemble des niveaux $e_{i}$ par rapport à leur position dans l'agrégat isolé (colonnes $\mathrm{A}_{1}-\mathrm{A}_{2}$ ).

2) L'enlèvement au voisinage immédiat de la cavité de 2 charges négatives produit une nouvelle descente des niveaux (colonnes $\mathrm{A}_{2}-\mathrm{A}_{3}$ ).

3) Cette descente s'accentue avec le nombre de charges négatives enlevées (colonnes $A_{3}-A_{4}$ ) près de la cavité.

4) Lorsque l'agrégat capte des charges négatives ( 2 ou 4 selon sa taille) l'ensemble des niveaux s'élève mais l'édifice demeure électroniquement stable (colonnes $A_{5}-A_{6}-A_{7}$ ). 

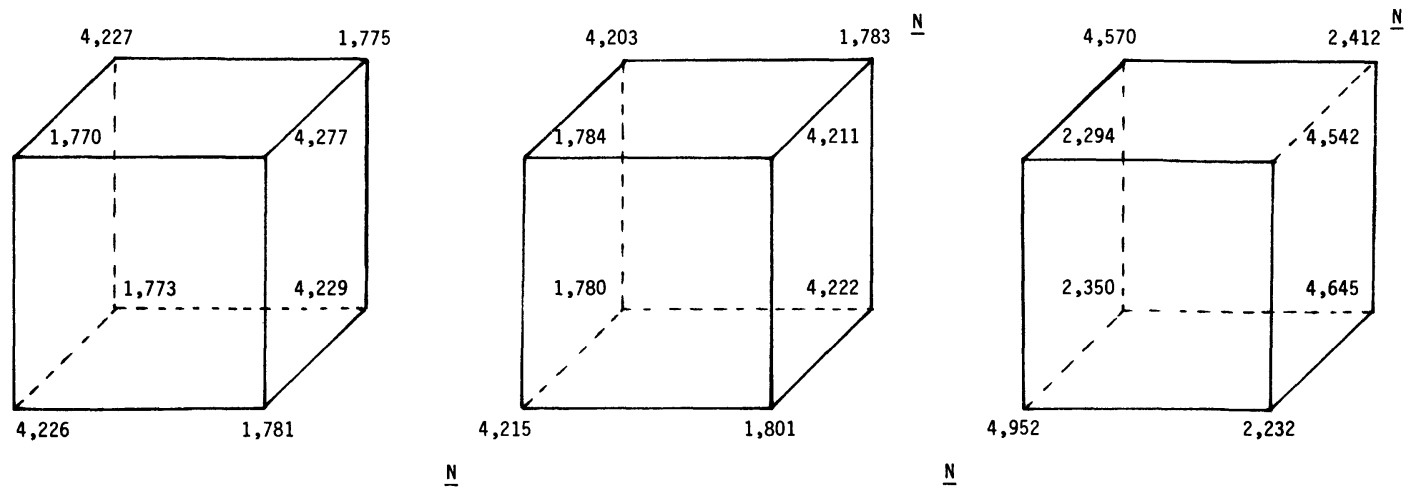

Fig. 2. - Répartition des charges sur un agrégat $\mathrm{Li}_{8}: \mathrm{A}:$ agrégat dans une cavité " neutre ». B : agrégat dans une cavité où on a enlevé 4 charges -. C : agrégat chargé 4 - dans une cavité où on a enlevé $4-$. $\mathrm{N}$ : emplacement des charges négatives enlevées dans le réseau au voisinage de l'agrégat.

[Repartition of charges on a $\mathrm{Li}_{8}$ aggregate : A : aggregate in a " neutral » cavity. B : aggregate in a cavity where four negative charges have been removed. $C$ : four negative charged aggregate - in a cavity where four negative charges have been removed. $\mathrm{N}$ : place of negative charges removed in the network near the aggregate.]

5) Si on enlève à la cavité « neutre » 2 charges positives, l'agrégat devient instable : le niveau HOMO est positif.

On a constaté d'autre part que l'orientation de la cavité selon un des axes 2 ou 4 du cube des charges n'avait qu'une influence minime sur les valeurs des niveaux électroniques donc sur les énergies de transition. Lorsque l'on considérait des cavités de plus en plus grandes on constatait comme il était prévisible, que la structure électronique se rapprochait de celle de l'agrégat isolé. En détruisant la symétrie cubique de l'ensemble des charges par addition d'une face de charges sur deux faces opposées du cube les niveaux électroniques varient très peu. En passant à des agrégats de taille supérieure $\left(\mathrm{Li}_{4}, \mathrm{Li}_{6}, \mathrm{Li}_{8}\right)$ on constate que les phénomènes conservent la même allure qualitative tout en diminuant d'ampleur.

On a aussi fait quelques calculs dans le cas du sodium, les phénomènes sont qualitativement les mêmes mais les amplitudes de variation sont beaucoup plus faibles.

En ce qui concerne les charges, elles sont également réparties sur les sommets lorsque l'agrégat est isolé. Il apparaît une dissymétrie lorsqu'on l'introduit dans la cavité. Si l'on caractérise cette dissymétrie par le rapport entre la charge la plus élevée et la charge la plus faible, on trouve $q=2,42$. Lorsqu'on enlève des charges négatives au voisinage de la cavité, ce rapport passe à $q=2,37$, il n'y a donc pas augmentation du moment électrique de $\mathrm{Li}_{8}$ par enlèvement de charges à son voisinage. On a $q=2,13$ si on a fixé 4 électrons sur l'agrégat.

3. Discussion. - La présence d'une matrice de charges autour de l'agrégat modifie donc sa structure électronique. Le travail de sortie se trouve augmenté.

On observe, dans tous les cas, un intervalle énergétique plus grand entre le HOMO et le LUMO (Lowest Unoccupied Molecular Orbital) qu'entre les niveaux vides successifs. L'introduction de 2 électrons sur le LUMO a pour effet de faire descendre ce niveau au voisinage de ce qui était précédemment le HOMO et de maintenir ainsi le gap entre les nouveaux HOMO et LUMO. Par exemple, dans le cas de $\mathrm{Li}_{2}$ on a :

$$
\begin{aligned}
& \Delta e=6,34 \mathrm{eV} \text { pour } \mathrm{Li}_{2} \text { isolé } \\
& \Delta e=6,99 \mathrm{eV} \text { pour } \mathrm{Li}_{2} \text { dans la cavité « neutre " } \\
& \Delta e=6,88 \mathrm{eV} \text { pour } \mathrm{Li}_{2} \text { dans une cavité où on a enlevé } 2 \text { charges }- \\
& \Delta e=7,48 \mathrm{eV} \text { pour } \mathrm{Li}_{2} \text { dans une cavité où on a enlevé } 4 \text { charges }-.
\end{aligned}
$$


Il ne semblerait donc pas que les propriétés optiques de l'agrégat doivent être beaucoup modifiées si l'on s'en tient à sa structure électronique mais il faut remarquer que les charges n'étant plus également réparties, il y a perte de symétrie, des transitions interdites deviennent donc permises ce qui ne peut manquer de se répercuter sur les propriétés optiques. Il est aussi important de remarquer qu'il peut fonctionner comme accepteur d'électrons ce qui est susceptible de modifier celles de la matrice qui l'entoure. Enfin, étant donné que la matrice de $\mathrm{MgO}$ a été représentée par un réseau de charges, les résultats précédents seront qualitativement valables pour toute matrice ionique.

\section{Bibliographie}

[1] Voir par exemple SeraPhim, B. O., Solar Energy Conversion, Topics in Applied Physics, 31, (Springer Verlag) 1979.

[2] Hehre, W. J., Lanthan, M. A., Dichtfield, R. D., Newton, Md., Pople, J. A., Program No 236, Quantum Chemistry Program Exchange, University of Indiana, Bloomington, Ind.

[3] Hehre, W. J., Communication privée.

[4] Julg, A. et Letoquart, B., Philos. Mag. 33 (1976) 721.

[5] Julg, A., Crystal as giant molecules, Lecture notes in Chemistry, Vol. 9, (Springer Verlag) 1978.

[6] Koopmans, T., Physica 1 (1934) 104.

[7] Arai, T., Sakamoto, M., J. Chem. Phys. 28 (1958) 32 et Rausil, B. J., J. Chem. Phys. 35 (1961) 669.

[8] Pour $\mathrm{Li}_{2}$, voir Kutzelnigg, W., Staemmler, V., Gelas, M., Chem. Phys. Lett. 13 (1972) 496. 